\title{
The use of Kubo formula to examine low temperature transport limited by interface roughness and phonons in metal-oxide-semiconductor field effect transistors
}

\author{
Yifei Zhang \\ Applied Physics Program, The University of Michigan at Ann Arbor, Ann Arbor, Michigan 48109-1120 \\ Jasprit Singh \\ Department of Electrical Engineering and Computer Science, The University of Michigan at Ann Arbor, \\ Ann Arbor, Michigan 48109-2122
}

(Received 3 August 1998; accepted for publication 9 November 1998)

\begin{abstract}
An approach to study transport in semiconductors using the Kubo formula is developed and applied to Si metal-oxide-semiconductor field effect transistors (MOSFETs). It is known that interface roughness is an important source of scattering in a MOSFET device operating at high sheet charge concentration $\left(\sim 10^{12} \mathrm{~cm}^{-2}\right)$. However, in spite of its importance, due to the complexity of the problem very simple models based on the Born approximation are used to study transport. The Born approximation breaks down when the interface quality is poor and transport occurs in lower energy localized states (for example, at low temperature). In this article we present results of a numerical method based on a three-dimensional approach to examine the interface roughness effects on electronic spectrum as well as on transport. Using the approach suggested by the Kubo formula we solve for the electronic states in the presence of interface roughness. Kubo formula is then used to study the transport properties as a function of sheet charge density, interface roughness level, and temperature. The model can easily be applied to other problems where scattering effects are very strong, e.g., in amorphous semiconductor devices. () 1999 American Institute of Physics. [S0021-8979(99)02904-7]
\end{abstract}

\section{INTRODUCTION}

Much of our understanding of transport in semiconductor devices depends upon the use of Born approximation and the independence of various scattering mechanisms. Scattering mechanisms such as ionized impurity scattering, interface roughness scattering, alloy scattering, etc. are handled within the Born approximation and are assumed to act independently. In many problems of interest it is known that Born approximation is not valid but it is still used because of the complexity of the problem. Examples include transport in metal-oxide-semiconductor field effect transistors (MOSFETs) at low temperature when a significant fraction of carriers are in the localized bandtail states, transport in clustered alloys, etc. The availability of powerful computers now makes it possible to address the transport problem using more accurate formalisms. The successful implementation of these more accurate formalisms can then allow us to address transport in materials like amorphous silicon, conducting polymers, clustered alloys, etc. In this article we present results on the use of Kubo formula in Si MOSFETs. We examine how interface roughness influences transport as the interface quality degrades.

In a MOSFET because of the lattice mismatch between $\mathrm{Si}$ and $\mathrm{SiO}_{2}$, the interface region has an amorphous nature with small but important distortions in the bond angles and bond lengths of the interfacial atoms which results in a rough $\mathrm{Si} / \mathrm{SiO}_{2}$ interface. ${ }^{1-4}$ It has been shown that the interface roughness is the major scattering source for a MOSFET device operating at high sheet charge concentration $\left(\sim 10^{12} \mathrm{~cm}^{-2}\right)$ resulting in the reduction of the electron mobility in the inversion layer of a MOSFET device. ${ }^{5-8}$ Therefore the quality of this $\mathrm{Si} / \mathrm{SiO}_{2}$ interface plays a critical role in the performance of MOSFETs. This role will become increasingly important as device dimensions shrink to below $0.1 \mu \mathrm{m}$ level. The reasons for this increasing importance are: (i) the random nature of the interface roughness will cause transport properties to fluctuate from device to device. For long devices the fluctuation effects are averaged out; (ii) the importance of localized states caused by interface roughness will increase as only tens of electrons are needed to switch the device; (iii) Born approximation which is the basis of current models to evaluate how interface roughness scattering influences transport will become invalid as the importance of localized states becomes more important.

In general when the disorder in a system is very small electronic states deviate only slightly from Bloch states. For small disorder the effect of the disorder can be accounted for fairly accurately within Born approximation. It has been shown ${ }^{9-11}$ that when disorder in a system increases low energy states can become localized while as the kinetic energy of the carriers increases the states become extended. The mobility edge separates the localized and extended states. We also know ${ }^{12}$ that localization due to disorder becomes more significant as the dimensionality of the system decreases. This is intuitively evident since with increasing dimension, the number of nearest neighbors in the solid increases and hence the pathways to move also increase. 
In the MOSFET it is known that the density of the twodimensional electron (hole) gas is described by bandtail states that arise from the disorder created by interface roughness. Transport in the MOSFET can be viewed as transport in the bandtail states and transport in the extended states where Born approximation is expected to hold. Transport in localized states is known to be described by phonon assisted variable range hopping where mobility increases with temperature. On the other hand in the extended states mobility decreases with temperature since both interface roughness and phonons reduce the coherence of the electronic states. In general in a MOSFET, transport occurs partially in the localized states and partially in extended states.

It is important to develop a formalism that can accurately describe transport regardless of the nature of the electronic state. Kubo formula is one such approach which in one limit is equivalent to the use of Born approximation and in the other limit can describe variable range hopping transport. The difficulty in using the Kubo formula is that the electronic problems (i.e., the wave functions and energy levels) have to be known in the presence of the disorder. While this has been very difficult to accomplish except in simple model systems, advances in computational resources have now made it possible to address realistic device structures. In this article we develop a three-dimensional formalism to address the MOSFET problem and use Kubo formula to find the conductivity (mobility) as a function of interface roughness and temperature.

The outline of the remainder of this article is as follows: in Sec. II, the modeling formalism is described in detail. In Sec. III, results of the formalism are discussed. The focus will be on the electronic spectrum, density of states, and the mobility in the inversion layer of Si MOSFET device and conclusions are made in Sec. IV.

\section{FORMALISM}

There are a number of important semiconductor devices where the nature of scattering is such that Born approximation becomes invalid. Examples are disordered semiconductor devices, devices in which alloy clustering is significant and silicon MOSFETs at low temperatures. New devices based on structures that have not yet been perfected (e.g., GaN/AlGaN transistors, oxide-GaAs heterostructures) also present situations where Born approximation becomes invalid. In general whenever wave function localization is significant (i.e., bandtail states are present) Born approximation becomes invalid.

In the case of Si MOSFET, perhaps the most important electronic device, interface roughness scattering is one of the most important scattering mechanisms. Models based on the Born approximation to study the interface roughness scattering have been used to study mobility in the two-dimensional channel. Using island height and lateral extent as fitting parameters, reasonably good agreement with experiments at room temperature ${ }^{13}$ has been demonstrated in high quality MOSFETs. However, at low temperatures it is known that localization effects due to interface roughness are quite important. Thus mobility increases with temperature instead of decreasing, a sign of hopping conduction. ${ }^{14-18}$ A formalism that can address transport in localized and extended states is the Kubo formula. However, to find the conductivity it is necessary to solve for a three-dimensional Hamiltonian including the interface roughness. The fast development of the modern computer allows us to do that now in a fairly small amount of time.

To calculate the electronic spectrum of a twodimensional electron gas in a MOSFET, two steps have been taken in this article. First, the potential profile along the direction perpendicular to the $\mathrm{Si} / \mathrm{SiO}_{2}$ interface in a MOS structure is obtained by solving the Schrödinger equation and Poisson equation self-consistently (one-dimensional problem). Then, the potential profile obtained in the onedimensional problem is used in a three-dimensional Schrödinger equation to yield the electron spectrum. In order to be consistent with the results obtained in the first step, the Fermi level of the MOSFET device is adjusted so that it gives the same occupation number.

Our model first gives the potential profile in a MOS structure by solving the Schrödinger equation and Poisson equation self-consistently. The Schrödinger equation yields the confined charge terms in the Poisson equation which, in turn, determines the potential profile. This potential profile is fed back into the Schrödinger equation until the solution of the Poisson equation goes to convergence. The onedimensional Poisson equation can be written as

$$
\frac{d^{2}}{d z^{2}} E_{c}(z)=-\frac{\rho(z)}{\epsilon(z)},
$$

where $E_{c}$ is the conduction band profile in the device, $\rho$ is the total charge density, and $\epsilon$ is the dielectric constant which can be changed in different regions of the device to account for different material parameters across interfaces. The total charge density $\rho$ is the sum of the doping charge, the free charge including the hole and electron, and the quantumconfined charge. This can be written as

$$
\begin{aligned}
\rho(z)= & q\left(N_{d}^{*}(z)-N_{a}^{*}(z)-n_{\mathrm{free}}(z)+p_{\mathrm{free}}(z)\right. \\
& \left.-\sum_{i} n_{i} \psi_{i}^{*}(z) \psi_{i}(z)\right)
\end{aligned}
$$

where $N_{a}^{*}$ and $N_{d}^{*}$ are the effective doping concentrations, $n_{\text {free }}$ and $p_{\text {free }}$ are the free carrier concentrations, and the sum is over $i$ two-dimensionally confined subbands of which normalized envelope functions are $\psi_{i}$ and in which the occupation is $n_{i}$ ( $p_{i}$ for the hole case). The effective doping concentrations, $N_{d}^{*}$ and $N_{a}^{*}$ can be written as

$$
\begin{aligned}
& N_{d}^{*}=N_{d}\left(\frac{1}{1+2 e^{\left(E_{f}-E_{d}\right) / k_{B} T}}\right), \\
& N_{a}^{*}=N_{a}\left(\frac{1}{1+4 e^{\left(E_{a}-E_{f}\right) / k_{B} T}}\right),
\end{aligned}
$$

where $N_{d}$ and $N_{a}$ are the concentrations of donor and acceptor dopants, and $E_{d}$ and $E_{a}$ are the impurity ionization ener- 
gies, respectively. The solution of the Poisson equation is performed by a vectorized Newton's method.

In the calculation of the charge density, one must determine the quantized two-dimensional charge and the free carrier charge. The quantized two-dimensional charge can be calculated from the eigenenergy levels obtained by solving the Schrödinger equation. The free carrier charge density can be written as

$$
n_{\text {free }}(z)=N_{c} F_{1 / 2}\left(\frac{E_{f}-E_{c}(z)}{k_{B} T}\right),
$$

where $N_{c}$ is the material effective density of states and $F_{1 / 2}$ is a half-order Fermi integral. The reason we use FermiDirac statistics instead of Boltzmann statistics, which have been used by many authors in the past, to determine the free carrier concentrations is that in heavily doped cases, the bands are near degenerate or degenerate, and Boltzmann statistics will overestimate the free carrier concentrations. To calculate the Fermi integral fast and accurately, a look-up table is used. Note that in Eq. (5), we use a threedimensional effective density of states to obtain the free carrier charge. However, to distinguish two-dimensional carriers with three-dimensional ones in the quantum well region, a cutoff value, which usually is set to be the lower boundary value, is used. For those carriers whose energy is below this cutoff value, they are treated as two-dimensional gas. For those whose energy is above this cutoff value, they are thus treated as free carriers.

In order to determine the two dimensionally confined charge profile, one must solve the Schrödinger equation for the subband envelope functions and their occupations. For the $n$-type MOSFET, the one-band Schrödinger equation can be used. The Schrödinger equation must, in general, be written in three dimensions, and the effective mass will be, in general, a tensor. In the electron case the $z$-dependent part of the Schrödinger equation is strictly separable from the inplane part of the equation which gives us extended Blochlike states. The one-dimensional ( $z$ dependent) Schrödinger equation can be written using the perpendicular part of the effective mass tensor as follows:

$$
\frac{d^{2}}{d z^{2}} \psi_{n}(z)+\frac{2 m_{w}}{\hbar^{2}}\left[E_{n}-V(z)\right] \psi_{n}(z)=0,
$$

where $m_{w}$ represents electron effective mass along the quantum confinement direction. This method is also referred to as the effective mass approximation method. For bulk silicon, the band edge of the conduction band is near $X$ point. Corresponding to this point there are six equivalent valleys. Under quantum confinement or strain effect, this sixfold degeneracy is decomposed into a twofold and a fourfold degeneracy. For electrons in the longitudinal valleys (along $\mathbf{k}_{\mathbf{z}}$ direction), $m_{w}$ is equal to $m_{l}$, electron longitudinal effective mass. Whereas for electrons in the transverse valleys (in-plane directions), $m_{w}$ is equal to $m_{t}$, electron transverse effective mass. Under the quantum confinement, the two longitudinal valleys will move down with respect to the four transverse valleys. This splitting is related to the difference of longitudinal and transverse effective mass. Since the lon- gitudinal effective mass for silicon is much larger than the transverse effective mass, the electron in the longitudinal valleys will occupy the lowest subband. In contrast, under the strain effect, the two longitudinal valleys will move up with respect to the four transverse valleys.

Once the Schrödinger equation has been solved for the envelope functions and the subband energy levels, it is straightforward to calculate the subband occupations. Since the subband density of states is constant with energy, the occupation comes from the first-order Fermi integral which is analytically integrable. In the electron case, we can write the occupation as:

$$
n_{i}=\frac{g_{i} m_{i} k_{B} T}{\pi \hbar^{2}} \ln \left[1+\exp \left(\frac{\left(E_{i}-E_{f}\right)}{k_{B} t}\right)\right],
$$

where $m_{i}$ and $g_{i}$ are the in-plane effective mass and the degeneracy of the $i$ th subband, respectively. In our case, for electrons in the longitudinal valleys, we have

$$
\begin{aligned}
& m_{i}=m_{t}, \\
& g_{i}=2 .
\end{aligned}
$$

Whereas for those in the transverse valleys, we have

$$
\begin{aligned}
& m_{i}=\left(m_{t} m_{l}\right)^{1 / 2}, \\
& g_{i}=4 .
\end{aligned}
$$

The reason we do not use $m_{l}$ as in-plane effective mass for electrons in the transverse valleys is because the in-plane mass tensor in this case has different values along $x$ and $y$ direction, which suggests an ellipsoidal shape for the constant energy surface.

After the one-dimensional potential is obtained from the first step, it is fed into the three-dimensional Schrödinger equation to yield the electronic spectrum with or without the presence of the interface roughness. The three-dimensional Schrödinger equation for the envelope functions, is given by

$$
\left(-\frac{\hbar^{2}}{2 m_{i}^{*}} \nabla^{2}+V(x, y, z)\right) \psi(x, y, z)=E \psi(x, y, z),
$$

where $V(x, y, z)$ is a three-dimensional potential incorporated with the interface roughness and $m_{i}^{*}(i=x, y, z)$ is the effective mass which is dependent on the direction. Like the onedimensional problem, the electrons in longitudinal and transverse valleys move with different effective masses therefore two sets of effective masses for electrons in longitudinal and transverse valleys are needed to calculate the electronic spectrum by solving the Schrödinger equation.

The rough interface is composed of islands of either the oxide region $\left(\mathrm{SiO}_{2}\right)$ or the channel region $(\mathrm{Si})$. These islands are randomly placed on the interface by a Monte Carlo method. If the island is $\mathrm{SiO}_{2}$, the potential is set to be the potential value of $\mathrm{SiO}_{2}$ at the rough interface whereas if the island is $\mathrm{Si}$, the potential is set to be the potential value of $\mathrm{Si}$ at the rough interface. The three-dimensional Schrödinger equation is solved by the finite-different technique. The boundary conditions along the $x y$ plane (parallel to the interface) are chosen to be periodic in order to calculate the transport quantities. The typical size of the matrix involved 
in our calculation is in the order of $10^{4} \times 10^{4}$. Since we solve for the electronic levels in the presence of the interface roughness in the MOS channel the effect of this roughness on the microscopic and macroscopic properties of the structure can be calculated directly from the electronic spectrum. We can thus answer the issues raised in Sec. I. To calculate the electron transport properties, such as conductivity and mobility, the Kubo formula ${ }^{19}$ has been used. The Kubo formula for the conductivity of electron under a small dc field is given by

$$
\sigma=\frac{2 \pi e^{2} \hbar^{3} V}{m^{* 2}} \int-\frac{\partial f}{\partial E}|D(E)|^{2} N^{2}(E) d E,
$$

where $V$ is the interface area and the reduced momentum matrix element $D\left(E, E^{\prime}\right)$ is expressed as

$$
D\left(E, E^{\prime}\right)=\int_{E=E^{\prime}} \psi_{E} \frac{\partial}{\partial x} \psi_{E^{\prime}} d \mathbf{r} .
$$

For a MOSFET device under the strong inversion, the electrons are confined in a narrow region $(100 \AA)$ of the channel near the interface. Equation (11) will render a conductance rather than conductivity. Once the conductance is derived, the mobility is readily calculated by the relation

$$
\mu=\frac{\sigma}{n e},
$$

where $n$ is the sheet charge density in the channel and $e$ is the electron charge.

To achieve reasonable results numerically calculated from a discrete electron spectrum due to the finite sample size chosen for study, a Gaussian broadening function is used for both the density of states, $N(E)$ and the reduced momentum matrix, $D(E)$. The reduced momentum matrix is evaluated by

$$
|D|_{\mathrm{av}}^{2}=\frac{\sum_{i}\left|\int \psi_{E_{i}}(\partial / \partial x) \psi_{E} d^{3} x\right|^{2} w_{\Gamma}\left(E_{i}, E\right)}{\sum_{i} w_{\Gamma}\left(E_{i}, E\right)},
$$

where $w_{\Gamma}\left(E_{i}, E\right)$ is an integral over the overlapping region between two Gaussian broadening functions centering on energy levels, $E_{i}$ and $E$, respectively. The quantity $\Gamma$ is the half width of the broadening functions and represents the lifetime of the corresponding state due to the effects of scatterings (phonons). Several groups have used the Kubo formula ${ }^{20-22}$ for studying transport at $0 \mathrm{~K}$. In these studies the conductivity is calculated at the limit of $\Gamma$ going to zero. To extend the Kubo formalism to finite temperature we use a phenomenological relation between scattering processes arising due to finite temperature and $\Gamma$. This is similar in spirit to the approach taken in deriving Mott variable range conductivity. This approach allows us to examine how phonon scattering and interface roughness scattering influence transport without treating them as independent scattering mechanisms. In this approach we view the total Hamiltonian as

$$
H=H_{\text {interface }}+H_{\text {phonon, }}^{\prime},
$$

where $H_{\text {interface }}$ is the Hamiltonian for the problem where interface roughness effects are included and results in localized eigenstates. The effect of $H_{\text {phonon }}^{\prime}$ is to cause a width to these eigenstates due to the vibration of the lattice. We evaluate this width by calculating the acoustic phonon scattering rates. ${ }^{23}$

In our model as temperature increases, two effects occur: (i) the carrier distribution spreads out due to the broadening of the Fermi function; (ii) the broadening of the individual eigenvalues occurs due to the increased phonon scattering. The outcome is that just as in the Mott variable range conductivity, mobility initially increases with temperature. This is because electrons localized at some region in space can now couple with more states. As the temperature increases to high values we expect that phonon scattering will eventually suppress mobility. It is important to note that in the Kubo formula, the electronic spectrum is obtained by solving the full Hamiltonian with the presence of interface roughness so that any correlation caused by the interface roughness is automatically included in the electronic spectrum.

\section{RESULTS AND DISCUSSION}

In order to solve the three-dimensional MOSFET problem it is important to choose a sample size that gives convergent results for density of states and mobility. The size depends on the interface quality, a more perfect interface requiring a larger size since the phase coherence length is larger. For the results reported here we have seen that in the channel plane (the $x y$ plane) a sample size of $1000 \AA \times 1000$ $\AA$ gives convergent results. In the $z$ direction since we are primarily interested in strong inversion cases, a sample size of $100 \AA$ is found to be adequate.

Along the $z$ direction the device structure is assumed to be made up of three regions: (i) a region which has perfect silicon-dioxide; (ii) a region that has a random distribution of $\mathrm{Si}$ and $\mathrm{SiO}_{2}$ islands; and (iii) a region that has perfect $\mathrm{Si}$. Interface roughness is introduced by randomly placing islands on the intermixed region of the MOS structure with island size given by

$$
\Delta L_{x}=25 \AA, \quad \Delta L_{y}=25 \AA, \quad \Delta L_{z}=5,10 \AA .
$$

Typical fluctuations generated by a random sequence on the interface region ( $x y$ plane) are shown in Fig. 1. The white regions represent material composed of $\mathrm{SiO}_{2}$ while the black regions represent material composed of $\mathrm{Si}$. We can see from Fig. 1 that large clusters of Si scatter on the interface and we expect that the localization if that occurs will be stronger in those cluster regions. We will focus on an energy region of about $30-40 \mathrm{meV}$ away from the band edge of Si conduction band since this is the region of interest for many devices.

The results shown in this section are for a MOSFET structure with $100 \AA$ oxide thickness and a background p-type doping of $1 \times 10^{14} \mathrm{~cm}^{-3}$.

Figures 2 and 3 show the electron probability distribution functions $P(x, y)$ on $x y$ plane in a MOSFET device operating at low and high sheet charge density (low and high voltage). The corresponding sheet charge densities are 1.5 $\times 10^{11}$ and $6.7 \times 10^{12} \mathrm{~cm}^{-2}$. The operating temperature is assumed to be 77 K. From Fig. 2, one can see that the electron wave functions for the device at low sheet charge density extend over the entire device as the case with a perfect 


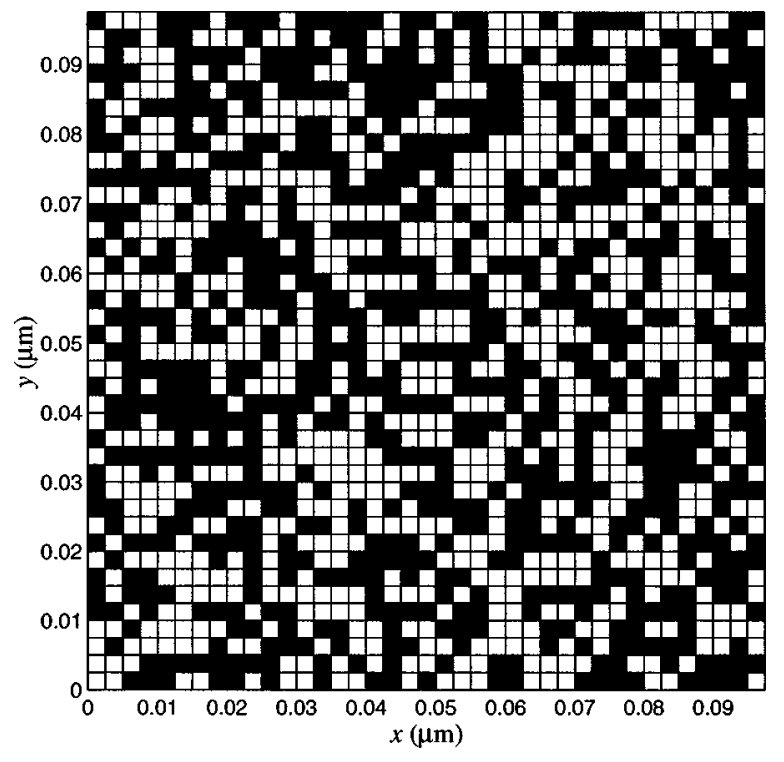

FIG. 1. Interface roughness generated by a random sequence. The black block represents $\mathrm{Si}$ material and the white block represents $\mathrm{SiO}_{2}$ material.

interface $^{24}$ and have a peak amplitude between 0.0015 and 0.004 corresponding to the normalization in the 1000 $\AA \times 1000 \AA x y$ plane. However, for the device working at high sheet charge density the eigenstates are quite different. We see from Fig. 3(a) that the low-lying states are strongly localized (e.g., the peak amplitude of the first state is 0.022 , one order of magnitude larger than the case at low sheet charge density). The lateral spread of these states is $200-250$ $\AA$. The position of these low-lying states will change if we change the random sequence of the disorder but the localization length is in the same range. This suggests that as one goes to the high sheet charge, the effect of interface roughness becomes stronger on the electronic states and thus the charge transport. To study transport in such a system the use of the Born approximation is invalid. We also see from Fig.

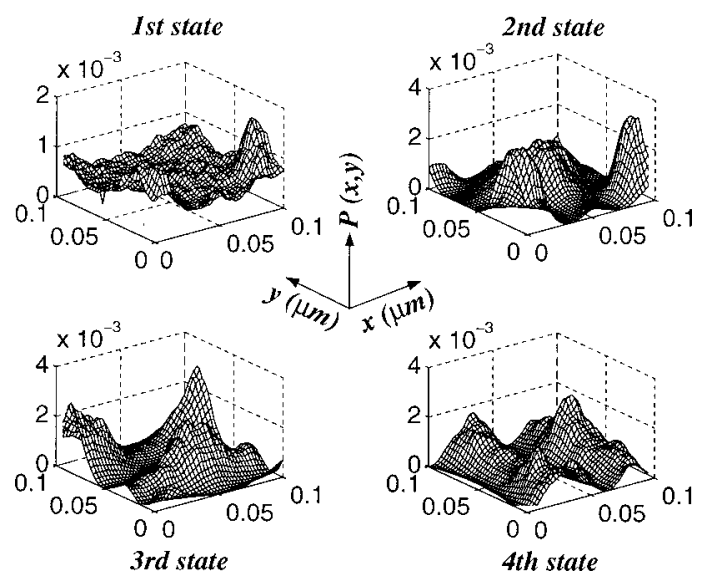

FIG. 2. Electron probability distribution functions $P(x, y)$ for a MOSFET device operating at low sheet charge density $\left(1.5 \times 10^{11} \mathrm{~cm}^{-2}\right)$ with an interface roughness (height: $5 \AA$, lateral extension: $25 \AA$ ). The wave functions extend to the device (chosen to be $0.1 \mu \mathrm{m} \times 0.1 \mu \mathrm{m}$ ). The MOSFET studied has 100 - $\AA$-thick $\mathrm{SiO}_{2}$ with the $p$-type substrate doped at $1 \times 10^{14} \mathrm{~cm}^{-3}$. The operating temperature is assumed to be $77 \mathrm{~K}$.
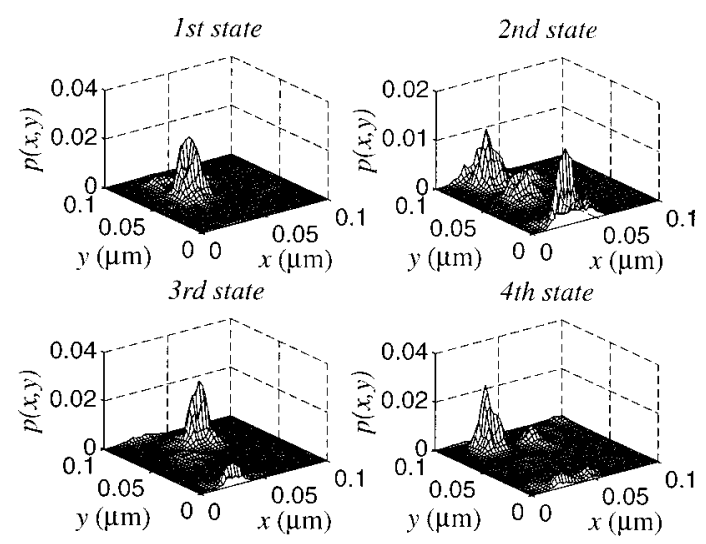

(a) Localized states

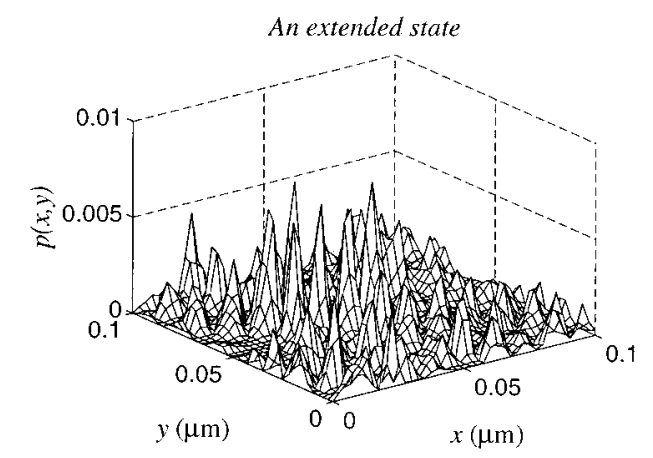

(b) Extended state

FIG. 3. Electron probability distribution functions $P(x, y)$ for the same interface roughness as Fig. 2 but operating at high sheet charge density ( 6.7 $\times 10^{12} \mathrm{~cm}^{-2}$ ). the low-lying states are clearly localized but the excited states are extended.

3(b) that as we go to higher energy states, the states become extended and spread over the entire structure.

We also find that interface roughness effects are strong under strong inversion conditions when the electrons are pushed close to the interface. When the device is biased near flatband conditions, the nature of the electronic wave functions shows that the wave functions are not localized and the use of Born approximation to address transport of carriers is quite valid.

The results for the density of states are shown in Fig. 4. We focus on the density of states near the band edges since these states are most influenced by interface roughness. The height of random islands at the interface, $\Delta L_{z}$, are chosen to be 5 and $10 \AA$. The density of states is calculated by using a $2.4 \mathrm{meV}$ Gaussian broadening function. As one can see from Fig. 4(a); interface roughness does not significantly alter the density of states in the system under flatband conditions. This is as expected intuitively since the electron wave functions are peaked away from the interfacial region. In contrast, as can be seen from Fig. 4(b), under the strong inversion the density of states is significantly affected by the interface roughness and bandtails are seen to develop as the interface roughness increases. This strong localization in the 


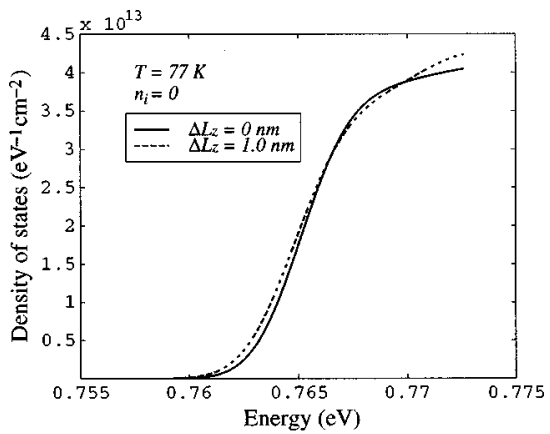

(a) Flat band

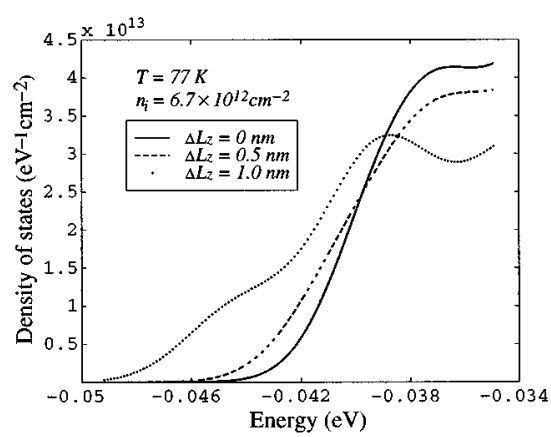

(b) Inversion

FIG. 4. Density of states functions under (a) flatband and (b) inversion conditions. Under strong inversion, the interface roughness causes significant bandtail states, especially for $\Delta L_{z}=1.0 \mathrm{~nm}$.

low-lying states can be attributed to the strong quantum confinement along the $z$ direction. This strong $z$ confinement leads to a large fraction of electrons occupying the interfacial region.

The bandtail width is shown in Fig. 5 as a function of interface roughness. The interface roughness goes from 0 to $10 \AA$. The bandtail width is obtained by measuring the width between the value of density of states at mobility edge and $e^{-1}$ of this value. The lower curve shows the bandtail width for the device under flatband condition. As one can see, the bandtail is almost independent of interface roughness. However, for the device under strong inversion, the bandtail width increases with interface roughness as shown in the

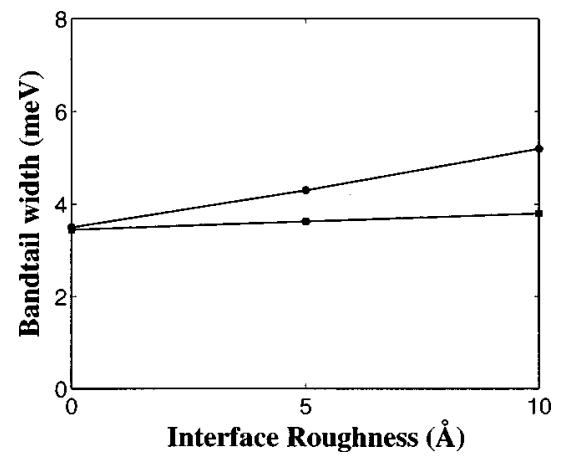

FIG. 5. Bandtail width vs interface roughness. Upper curve is for device under strong inversion and lower curve is for device under flatband condition. The bandtail width is independent of interface roughness for device under flatband condition and increases with interface roughness for device under strong inversion.

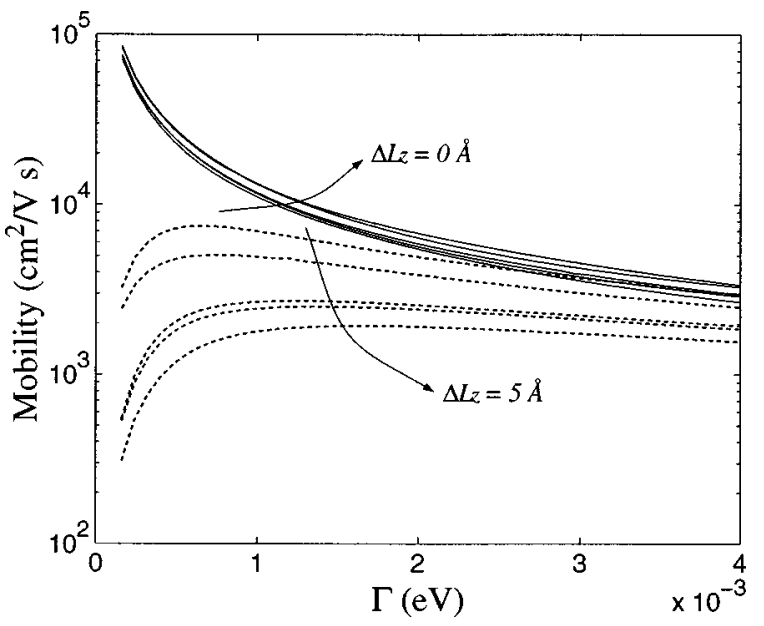

FIG. 6. Electron mobility as a function of $\Gamma$. Solid curves and dotted curves represent mobilities for the device with a perfect interface and roughness interface: $\Delta L_{z}=5 \AA$, respectively. The corresponding sheet charge densities in the channel from the bottom dotted curve to the top dotted curve are $(6.7$, 5.6, 4.6, 3.6, and 2.6) $\times 10^{12} \mathrm{~cm}^{-2}$. The device under study has a dimension of $500 \AA \times 500 \AA \times 100 \AA$.

upper curve. At zero interface roughness, the bandtail width is not zero. This is caused by the finite sample size and the broadening effect.

Next we report results for the mobility in the channel using the Kubo formula. From our discussion of localization we can see that the imperfect interface mobility will be very small unless electrons can couple from one state to another by other scattering processes such as phonons. Thus for the imperfect interface case we expect that as the broadening parameter $\Gamma$ increases, mobility will initially increase. For the case of the perfect interface, on the other hand, as $\Gamma$ increases the mobility should decrease.

Figure 6 shows results for channel mobility as a function of $\Gamma$ for MOSFET device. Results for the perfect interface are given by solid curves and while the dashed curves represent the results for the rough interface. The device is assumed to be under inversion (sheet charge density of 6.7 $\times 10^{12} \mathrm{~cm}^{-2}$ ) operating at $77 \mathrm{~K}$. As one can see from the figure, the mobilities (solid curves) for the MOSFET device with a perfect interface decrease with $\Gamma$ as expected. However for the case with interface roughness, the dependence of mobility on $\Gamma$ is totally different. Initially the mobility increases but when $\Gamma$ becomes large mobility decreases.

For the MOSFET at $77 \mathrm{~K}$ acoustic phonon scattering is expected to be the dominant scattering mechanism. As can be seen from Fig. 6, the phonon scattering actually assists the conduction in the inversion layer for the device with interface roughness at low $\Gamma$ value which results in an increase in the mobility at small $\Gamma$. This is observed in the experiment and becomes a signature of the variable range hopping conductivity. ${ }^{15-18}$ Using the acoustic phonon scattering rates for the $\mathrm{Si}$ inversion layer we find that $\Gamma$ is expected to be $0.34 \mathrm{meV}$.

In Fig. 7 we show the mobility for the perfect interface and for the rough interface with $\Delta L_{z}=5 \AA$ as a function of the sample size along the interface. The MOSFET under study is under strong inversion with the sheet charge density 


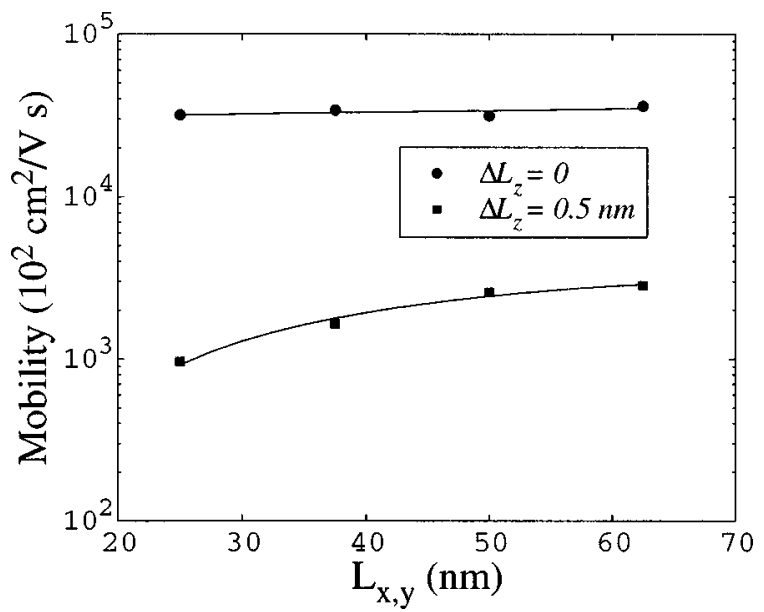

FIG. 7. Electron mobility as a function of the sample size, $L_{x, y}$ along the interface for (a) $\Delta L_{z}=0 \mathrm{~nm}$ and (b) $\Delta L_{z}=0.5 \mathrm{~nm}$. The MOSFET under study is under strong inversion with the sheet charge density of 4.6 $\times 10^{12} \mathrm{~cm}^{-2}$. The half width of Gaussian broadening function, $\Gamma=0.34$ $\mathrm{meV}$ corresponding to the phonon scattering rate at $77 \mathrm{~K}$.

of $4.6 \times 10^{12} \mathrm{~cm}^{-2}$. The mobility for the perfect interface is almost independent of the sample size due to the extended nature of the electronic wave functions. In contrast, the mobility for the rough interface increases with the sample size. This is because the spatial extent of the localized wave function is comparable with the sample size. However, we found the sample size of $500 \AA \times 500 \AA$ along the $x y$-plane was adequate to yield convergent results as shown in Fig. 7. So, in the following studies, we will use the sample size of 500 $\AA \times 500 \AA$ for all the mobility calculations.

In Fig. 8 we show the calculated relationship between the mobility and sheet charge density in the channel. Results are shown for the perfect interface and for imperfect interfaces with $\Delta L_{z}=5 \AA$ and $\Delta L_{z}=10 \AA$. From the figure, one can see that the mobility for the device with perfect interface is almost independent of sheet charge density. This value agrees quite well with mobility calculated using Born ap-

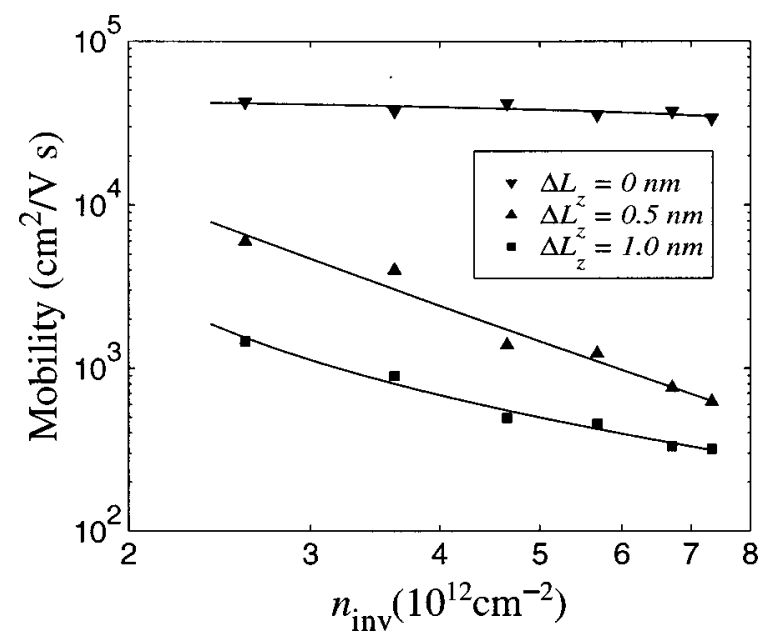

FIG. 8. Electron mobility as a function of sheet charge density, $n_{\text {inv }}$ for (a) $\Delta L_{z}=0 \mathrm{~nm}$, (b) $\Delta L_{z}=0.5 \mathrm{~nm}$, and (c) $\Delta L_{z}=1.0 \mathrm{~nm}$. The half width of Gaussian broadening function, $\Gamma=0.34 \mathrm{meV}$ corresponding to the phonon scattering rate at $77 \mathrm{~K}$.

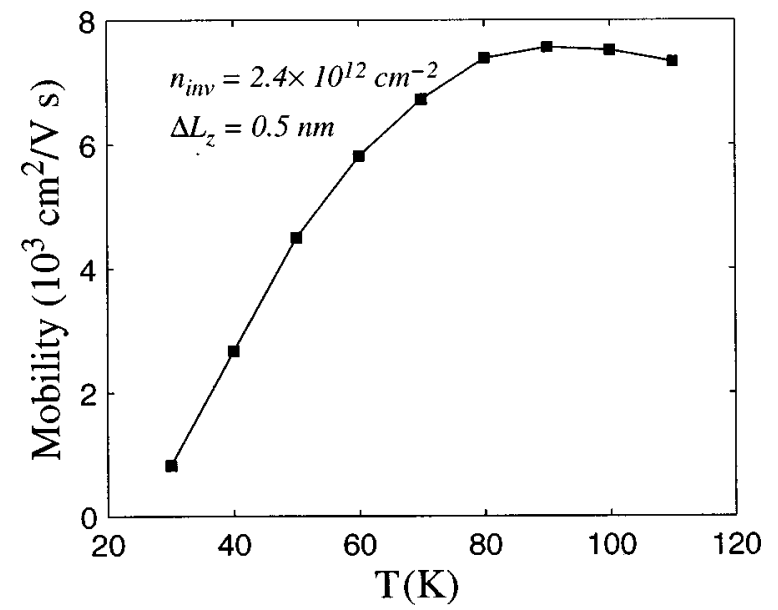

FIG. 9. Electron mobility as a function of temperature, $T$. The corresponding sheet charge density is $2.4 \times 10^{12} \mathrm{~cm}^{-2}$.

proximation and acoustic phonon scattering. For the device with the interface roughness, mobility is found to have a strong dependence on sheet charge density. This is to be expected since as the sheet charge density is increased the electron charge is pushed closer to the interface. Use of the Born approximation shows that mobility should be inversely proportional to the square of the sheet charge. For the case with $\Delta L_{z}=5 \AA$, we find that the mobility is proportional to $n_{\text {inv }}^{-2.2}$, a slight deviation from the model based on the Born approximation. For the case of $\Delta L_{z}=10 \AA$ where localization effects are very strong, there is no simple relation between the mobility and the sheet charge density.

In Fig. 9 we show the mobility as a function of temperature from 30 to $110 \mathrm{~K}$ for $\Delta L_{z}=5 \AA$. The corresponding sheet charge density is $2.4 \times 10^{12} \mathrm{~cm}^{-2}$. Figure 9 shows that the mobility increases as temperature increases at low temperature. As discussed earlier this is attributed to the importance of phonon-assisted hopping in this regime. It is not possible to understand this regime using Born approximation and the standard transport theory where scattering mechanisms are independent. However, as the temperature increases to high enough, the mobility starts to decrease as shown in Fig. 9. This is primarily due to the increasing phonon scattering as the temperature increases. This characterization of electron mobility has been verified in the experiment. $^{25}$

\section{CONCLUSION}

In this article we have developed a formalism that allows us to study transport without relying on Born approximation and independent scattering approximation. The approach is based on three-dimensional solutions of the Schrödinger equation in the presence of the disorder potential. The feasibility of the method based on the Kubo formula has been demonstrated by applying it to a Si MOSFET under a range of biasing conditions. Mobility has been evaluated under conditions where a significant fraction of states are localized. The model allows us to examine transport in the variable range hopping regime where mobility increases with temperature. We have also seen that the exact dependence of 
mobility in a MOSFET on sheet charge deviates from the $\mu \propto n_{\text {inv }}^{-2}$ relation. The generality of the model presented makes it a useful method to study transport in devices where disorder is severe, e.g., in amorphous Si transistors-devices where Born approximation completely breaks down.

\section{ACKNOWLEDGMENTS}

This work was funded by U.S. Air Force (Grant No. AFOSR-91-0349) and the U.S. Army Research Office.

${ }^{1}$ F. J. Grunthaner, P. J. Grunthaner, R. P. Vasquez, B. F. Lewis, J. Moserjion, and A. Madhukar, J. Vac. Sci. Technol. 16, 1443 (1979).

${ }^{2}$ S. M. Goodwick, D. K. Ferry, C. W. Wilmsen, Z. Liliental, D. Fathy, and O. L. Krivanek, Phys. Rev. B 32, 8171 (1985).

${ }^{3}$ R. S. Bauer, R. Z. Bachrach, and L. J. Brillson, Jpn. J. Appl. Phys., Part 1 33, 221 (1994).

${ }^{4}$ T. Yoshinobu, A. Iwamoto, and H. Iwasaki, Jpn. J. Appl. Phys., Part 1 33, 383 (1994).

${ }^{5}$ Y. C. Cheng and E. A. Sullivan, Surf. Sci. 34, 717 (1973).

${ }^{6}$ T. Ando, J. Phys. Soc. Jpn. 43, 1616 (1977).

${ }^{7}$ T. Hori and H. Iwasaki, IEEE Electron Device Lett. 10, 195 (1989).
${ }^{8}$ G. H. Kruithof, T. M. Klapwijk, and S. Bakker, Phys. Rev. B 43, 6642 (1991).

${ }^{9}$ P. W. Anderson, Phys. Rev. 109, 1492 (1958).

${ }^{10}$ N. F. Mott, Metal-Insulator Transition (Taylor and Francis, London, 1974).

${ }^{11}$ P. A. Lee and T. V. Ramakrishnan, Rev. Mod. Phys. 57, 287 (1985).

${ }^{12}$ E. Abrahams, P. W. Anderson, D. C. Licciardello, and T. V. Ramakrishnan, Phys. Rev. Lett. 42, 673 (1979).

${ }^{13}$ S. Yamakan, H. Ueno, K. Taniguchi, and C. Hamaguchi, J. Appl. Phys. 79, 911 (1996).

${ }^{14}$ N. F. Mott, Electron. Power 19, 321 (1973).

${ }^{15}$ M. Pepper, S. Pollitt, and C. J. Ankins, Phys. Lett. A 48, 113 (1974).

${ }^{16}$ M. Pepper, S. Pollitt, and C. J. Ankins, J. Phys. C 7, L273 (1974).

${ }^{17}$ D. C. Tsui and S. J. Allen, Phys. Rev. Lett. 32, 1200 (1974).

${ }^{18}$ D. C. Tsui and S. J. Allen, Phys. Rev. Lett. 34, 1293 (1975).

${ }^{19}$ See, for example, S. Doniach and E. H. Sondheimer, Green's Functions for Solid State Physicists (W. A. Benjamin, Reading, MA, 1974); D. K. Ferry, Semiconductors (Macmillan, New York, 1991).

${ }^{20}$ J. Stein and U. Krey, Z. Phys. B 37, 13 (1980).

${ }^{21}$ D. J. Thouless and S. Kirkpatrick, J. Phys. C 14, 235 (1981).

${ }^{22}$ S. Yoshino, in Anderson Localization, edited by Y. Nagaoka and H. Fukuyama (Springer, Berlin, 1982), p. 68.

${ }^{23}$ J. Singh, Physics of Semiconductors and their Heterostructures (McGrawHill, New York, 1993).

${ }^{24}$ Y. Zhang and J. Singh, Appl. Phys. Lett. 73, 1577 (1998).

${ }^{25}$ F. F. Fang and A. B. Fowler, Phys. Rev. 169, 619 (1968). 